Authors' reply: The letter from Rodger et al uses our editorial to rehearse the well-worn arguments of the small group of so-called 'critical psychiatrists' who are active and vocal in criticising core aspects of the practice of psychiatry as a medical subspecialty underpinned by science. The views expressed in the letter are mainly tangential to the views we expressed in our editorial and the authors have made assumptions and accusations that are unsupported by our text.

We are very keen to encourage informed and constructive debate to advance patient care and mental health. However, it is important to make a distinction between the freedom that is properly enjoyed in academic debate and the responsibilities that come with professional practice. At present, those who work as psychiatrists are expected to practise in accordance with evidence-based standards. The standards we adhere to will of course change over time as the evidence base develops. This is expected by patients and colleagues and required by regulators.

We continue to believe that our patients are best served by seeing psychiatrists who are trained to make a thorough assessment, come to a diagnosis and shared formulation with the patient of their problems and use this to draw up an evidence-based management plan. It seems strange to us that this should be surprising, contentious or upsetting to the authors of the letter.

Nick Craddock, Department of Psychological Medicine and Neurology,

school of Medicine, Cardiff University, email: craddockn@cardiff.ac.uk;

Laurence Mynors-Wallis, Alderney Hospital.

doi: 10.1192/bjp.205.6.498

\section{Concerns regarding an evaluation of MTFC-A for adolescents in English care}

We are writing to highlight concerns regarding conclusions offered by Green et al in their evaluation of Multidimensional Treatment Foster Care for Adolescents (MTFC-A) relative to usual care for at-risk youth in English foster care. ${ }^{1}$ We commend the authors for undertaking an independent review of MTFC-A. However, we offer some observations to help contextualise the efficacy of the evaluation with respect to the primary conclusion that MTFC-A did not result in better outcomes than usual care.

Green et al's evaluation employed a two-arm, single-blinded (assessor) randomised controlled trial embedded within an observational quasi-experimental case-control study. An intentto-treat (ITT) analysis was employed specific to the MTFC-A versus usual care comparison. The authors state that the study was intended to be powered at $\beta=0.80$ to detect half a standard deviation difference between ITT and usual care (with a target $n$ of 130), and was powered $\beta=0.95$ to detect the same effect between ITT and usual care in the quasi-experimental study (with a target $n$ of 90). However, the target allocation for the trial was not met. The trial randomly allocated only 34 participants ( $n=20$ MTFC-A and $n=14$ usual care). Based on these numbers, we estimate the study was actually powered at $\beta=0.29$ in the ITT analysis to detect half a standard deviation difference between conditions assuming equal variances, and at $\beta=0.28$ assuming unequal variances.

Substantive conclusions therefore seem to be based on a substantially underpowered design (as far as we can tell from the detail presented in the original manuscript). Further, the quasi-experimental arm was described as a case-control design. However, it was not a matched case-control design. This is evident from multiple baseline differences between groups, some of which remained after an intensive set of propensity-score weights was applied and after elimination of cases with probability of assignment to MTFC-A above 0.95 and below 0.05. Depending on the distribution of assignment probabilities, this may have resulted in relatively limited 'data trimming' in order to attain desired allocation probabilities near 0.50 . The observed differences included not only age but also the primary outcome scores.

Notwithstanding concerns regarding statistical power for the trial, the authors reported intervention by baseline risk interactions in the only adequately powered arm of the study (see Table 5). Given prior demonstration of MTFC-A intervention by baseline risk interactions, ${ }^{2}$ these results may have been more appropriately presented as a hypothesised replication. Statistical power is also a concern for the reported analyses of offending; $\beta=0.034$ to detect the observed ITT odds ratio of 1.24 using an allocation of 20 and 14 cases, and $\beta=0.031$ in the quasiexperimental arm to detect the observed ITT odds ratio of 1.07 with 93 and 92 cases. Interpretation of effects should therefore be treated with caution.

We raise one additional point of clarification regarding prior MTFC-A implementations. The authors state that the context of intervention in the UK differs significantly from that in the originating US studies, since 'these were focused on convicted delinquent youth where the alternative [to MTFC-A] was incarceration', thereby concluding that the 'control condition in the US studies approximated [...] to juvenile custody'. Actually, similar to the usual care condition in the Green et al study, the standard control condition in US MTFC-A studies is group care, ${ }^{3}$ not incarceration.

We offer these points by way of lending interpretation to the efficacy of Green et als results and to suggest caution in accepting the conclusion that MTFC-A may not result in better outcomes than usual care among at-risk adolescents in English care.

1 Green JM, Biehal N, Roberts C, Dixon J, Kay C, Parry E, et al. Multidimensional Treatment Foster Care for Adolescents in English care: randomised trial and observational cohort evaluation. Br J Psychiatry 2014; 204: 214-21.

2 Leve LD, Chamberlain P, Smith DK, Harold GT. Multidimensional treatment foster care as an intervention for juvenile justice girls in out-of-home care. In Delinquent Girls: Contexts, Relationships, and Adaptation (eds S Miller, L Leve, P Kerig). Springer Press, 2011.

3 Chamberlain P. Treating Chronic Juvenile Offenders: Advances Made through the Oregon Multidimensional Treatment Foster Care Model. American Psychological Association, 2003.

Conflict of interest: The authors have collaborated with US colleagues on projects using the MTFC-A programme.

Gordon T. Harold, School of Psychology, University of Sussex. Email: g.harold@sussex.ac.uk; David S. DeGarmo, Prevention Science Institute, University of Oregon.

doi: $10.1192 /$ bjp.205.6.498a

Authors' reply: Harold \& DeGarmo correctly refer to points regarding sample size and power that we already made in the discussion section of our paper. Despite this, we did point to the strengths of the study in the representativeness of the cohort within a real-world implementation setting, the fact that the study was conducted independently of treatment originators and UK implementation team, careful attention to triangulation and masked rating of primary outcome data (something often not undertaken in this kind of context), and the low attrition rates to endpoint. We stated that the convergence of findings from our mixed-method design and the confidence intervals of the outcome estimations gave some confidence to inferences from the results. 